\title{
The Influence of Hearing Aids on Balance Control: A Systematic Review
}

\author{
Daniele Borsetto $^{a}$ Virginia Corazzi ${ }^{b}$ Sebastiano Franchellac, $d$ \\ Chiara Bianchini $^{b}$ Stefano Pelucchi ${ }^{b}$ Rupert Obholzer ${ }^{a}$ Andrew J. Soulby ${ }^{a}$ \\ Nikul Amin ${ }^{a}$ Andrea Ciorbab \\ aENT Department, Guy's and St Thomas' Hospitals, London, UK; ${ }^{b}$ ENT \& Audiology Department, University Hospital \\ of Ferrara, Ferrara, Italy; 'Department of Neurosciences, Otolaryngology Section, University of Padua, Padua, Italy; \\ dDepartment of Woman's and Child's Health, University of Padova, Padova, Italy
}

\section{Keywords}

Hearing aid · Balance · Dizziness · Postural control ·

Vestibular diseases . Peripheral vestibular loss · Systematic review

\begin{abstract}
Aim: To assess the current opinion on the effects of hearing loss treatment by hearing aids (HAs) and the benefits of HA use on imbalance. Methods: PRISMA-compliant systematic review was done, including observational studies in patients affected by mild to severe sensorineural hearing loss with $\mathrm{HAs}$, investigating the benefits of HAs on balance. Electronic searches were performed through Medline, Cochrane, Embase, Web of Science, and Scopus. Results: A total of 200 patients in 8 studies were included in this systematic review. Four studies were cross-sectional, 3 cross-sectional controlled and 1 prospective nonrandomized study. Static and dynamic balance in the aided condition improved in patients assessed using clinical investigations including Romberg test and Functional Ambulation Performance/miniBESTest, respectively. Variable outcomes were found mea-
\end{abstract}

suring static and dynamic balance during the aided condition with objective tests (computerized posturography, Mobility Lab device). Improved quality of life outcomes and self-confidence were noted, while subjective measurements of balance had conflicting results. Conclusion: Although an improvement in balance in patients with HAs has been shown in certain conditions, the overall benefit is still unclear and it is only possible to speculate that HAs may also improve static, dynamic, or subjective perception of balance function in adults affected by hearing loss.

๑) 2020 S. Karger AG, Basel

\section{Introduction}

Hearing loss is one of the most common sensory deficits affecting $>5 \%$ of the world's population. In Europe, 52 million people aged over 65 years $(10 \%$ of the entire

Daniele Borsetto and Virginia Corazzi contributed equally to the paper.

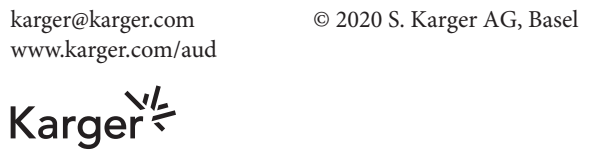

Sebastiano Franchella

Department of Neurosciences, Otolaryngology Section Department of Woman's and Child Health, University of Padova Via Giustiniani 2, IT-35100 Padova (Italy)

sebastiano.franchella@unipd.it 
population) self-report hearing loss [EuroStat, 2015] with approximately $90 \%$ of elderly people over 80 years old being affected [Lacerda et al., 2012]. Hearing loss has been shown to be associated with multiple comorbidities, including poor physical health, anxiety, depression, isolation, cognitive decline, and dementia [Access Economics, 2006; Hogan et al., 2009; Gates et al., 2010; Huang et al., 2010; Deal et al., 2015; Taljaard et al., 2016; Loughrey et al., 2018; Mick et al., 2018]. Moreover, hearing loss is often associated with imbalance with suggested theories including (i) age-related loss of labyrinthine function, in which hearing loss is a surrogate marker for vestibular hypofunction leading to imbalance or (ii) postural stability is maintained by sensory information from visual, audio-vestibular, and somatosensory inputs and therefore loss of audiological cues in patients with hearing loss directly impacts on balance [Viljanen et al., 2009; Rumalla et al., 2015].

The objective effects of hearing aids (HAs) on balance in patients affected by hearing loss are yet to be examined in a large comprehensive randomized study. The assumption that auditory rehabilitation of patients with hearing loss will improve their spatial cues, important with postural control, has primarily been investigated in patients with severe-to-profound hearing loss receiving cochlear implants (CI) [Ibrahim et al., 2017]. The findings from this meta-analysis suggest that CI leads to variable effects on the balance depending on age at implantation and etiology of the sensorineural hearing loss (SNHL) [Ibrahim et al., 2017].

Considering that every year, one-third of communitydwelling elderly patients over 65 will have a fall and that the complications due to these falls are associated with a $17 \%$ all-cause mortality risk within 6 months, it is very important to explore the role that hearing loss and rehabilitation has on balance[Lord et al., 2003].

Static measures of balance, by quantifying stability while standing still, have been linked to imbalance/fall risk [Piirtola and Era, 2006; Swanenburg et al., 2010]. However, gait performance is at least as important as static balance and decreases in dynamic balance performance (effective balance function under dynamic conditions), especially gait, are linked to increased imbalance and risk of falling [Verghese et al., 2009; Callisaya et al., 2012; Thaler-Kall et al., 2015]. We present the first systematic review of the effects of HAs in patients with mild to severe hearing loss on balance and evaluate the potential benefit of auditory amplification on balance, in patients with hearing loss.

\section{Methods}

\section{Outcome Measures}

Primary Outcome Measures

1. a. Clinical investigations on

i. Static balance determined through measures such as Romberg (on foam or/and tandem stance).

ii. Dynamic balance determined through measures such as Functional Ambulation Performance (FAP), Timed Up and Go (TUG), and Mini-BESTest.

b. Objective measures of

i. Static balance determined through measures such as computerized posturography.

ii. Dynamic balance determined through measures such as computerized posturography and inertial sensors (e.g., Mobility Lab).

Secondary Outcome Measures

2. a. Quality of life (QoL), measured using validated instruments such as SF-36 (Short-Form Health Survey Questionnaire) and SADL (Satisfaction with Amplification in Daily Life).

b. Subjective balance, measured using validated instruments with adequate psychometric properties such as FES-I (Falls Efficacy Scale-International), BBS (Berg Balance Scale) and ABC (Activities-specific Balance Confidence) Scale.

3. Adverse outcomes related to use of hearing aid(s).

\section{Search Strategy}

We searched published, unpublished, and ongoing studies through Medline, Cochrane, Embase, Web of Science, and Scopus evidence from inception through to April 27, 2020. The research was conducted according to PRISMA criteria [Moher et al., 2015].

Search strategies were modeled on the MEDLINE search terms described below. Prior to searching the databases, the search terms were peer reviewed to ensure that they conformed to PRESS guidelines (https://www.sciencedirect.com/science/article/pii/S0895435616000585), and the search syntax was adapted where necessary.

The search strategies used were hearing aid OR hearing aids OR implant OR hearing implant.mp ( $\mathrm{mp}=$ title, abstract, original title, name of substance word, subject heading word, floating subheading word, keyword heading word, organism supplementary concept word, protocol supplementary concept word, rare disease supplementary concept word, unique identifier, and synonyms), AND vestibular OR caloric OR VEMP OR balance OR posturography OR dizziness OR Dizziness Handicap Inventory OR Romberg OR landmark OR posture OR stability OR Spatial orientation OR Stabilometry OR Postural sway OR Sensory reweighting OR Biodex balance system OR Fall risk test.mp. The reference lists of identified articles were reviewed to identify any additional studies for inclusion.

\section{Exclusion Criteria}

Exclusion criteria included studies published in non-English languages and studies containing aggregated or duplicated data from previously published work.

\section{Selection of Studies and Data Extraction}

Two authors (DB and SF) independently screened all titles and relevant abstracts from the search strategy and evaluated the full 
Fig. 1. Flow chart of study inclusion pro-

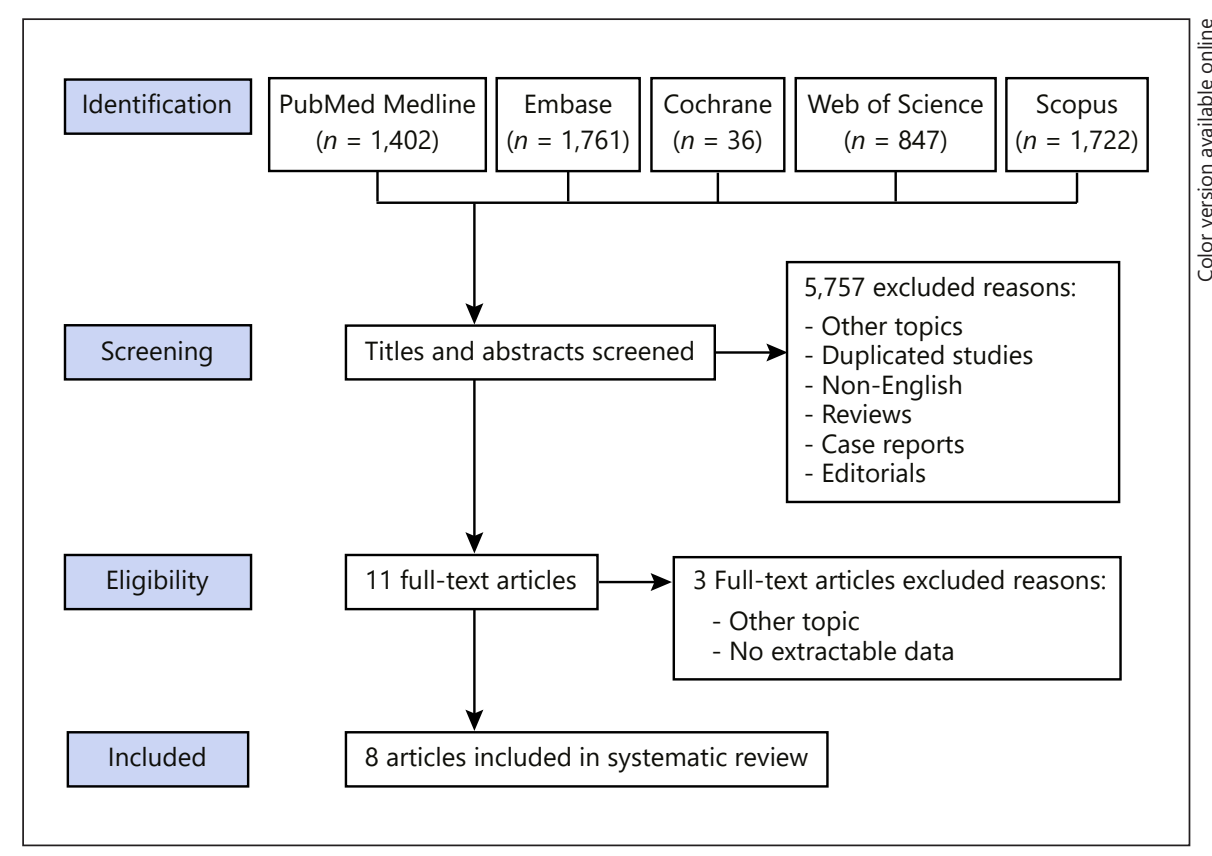

texts of relevant articles against the inclusion criteria (Fig. 1). Any disagreement between the assessors on the suitability of articles for inclusion was settled through consensus, and persistent disagreement referred to the senior author (AC).

Data from included studies were extracted using a standardized pro forma. Data extracted at the study level included author, year of publication, article language, and number of cases. Data extracted at the patient level included sample size, demographics, diagnosis, measurements (questionnaires and tests administrated), and outcome measures.

Data Analysis

Due to the heterogeneity in presentation of data, a statistical meta-analysis of these results was not possible, and the results were presented descriptively.

Risk of Bias and Quality Assessment

Studies were assessed for quality and risk of bias by both reviewers, according to a modification of the system described in the Cochrane Handbook for Systematic Reviews of Interventions (V5.1.0.180).

\section{Results}

\section{Result of Search Strategy and Description of Studies}

The search identified 5,768 articles. After screening, we identified 11 potentially eligible studies and undertook a full text review. Three studies did not meet the inclusion criteria, and 8 eligible studies were included in the review (Fig. 1).
The 8 studies included 4 cross-sectional studies [Lacerda et al., 2012; Rumalla et al., 2015; Shayman et al., 2017; McDaniel et al., 2018], 3 cross-sectional controlled studies [Vitkovic et al., 2016; Negahban et al., 2017; Maheu et al., 2019], and 1 prospective nonrandomized study [Weaver et al., 2017]. The characteristics of included studies are demonstrated in Table 1.

\section{Participants}

A total of 200 patients were included in the systematic review. The mean age was $67.5 \pm 10.6(\mathrm{SD})$ years (range 19-95). The male:female ratio was 1:1. Hearing threshold data were documented in 6 out of 8 studies ( 173 subjects out of 200) with 9 patients affected by mild SNHL, 104 patients by moderate SNHL, 40 patients by severe SNHL, and 20 patients by profound SNHL. A total of 138 patients were HAs users. Ten out of 138 patients from one study were diagnosed with both SNHL and balance problems, documented by vestibular objective measurements (such as video head impulse test, caloric examination, cervical and ocular vestibular evoked myogenic potentials) [Maheu et al., 2019]. Twenty-five patients with hearing loss but non-HAs users were considered as a part of a control cohort only in one study [Negahban et al., 2017]. 


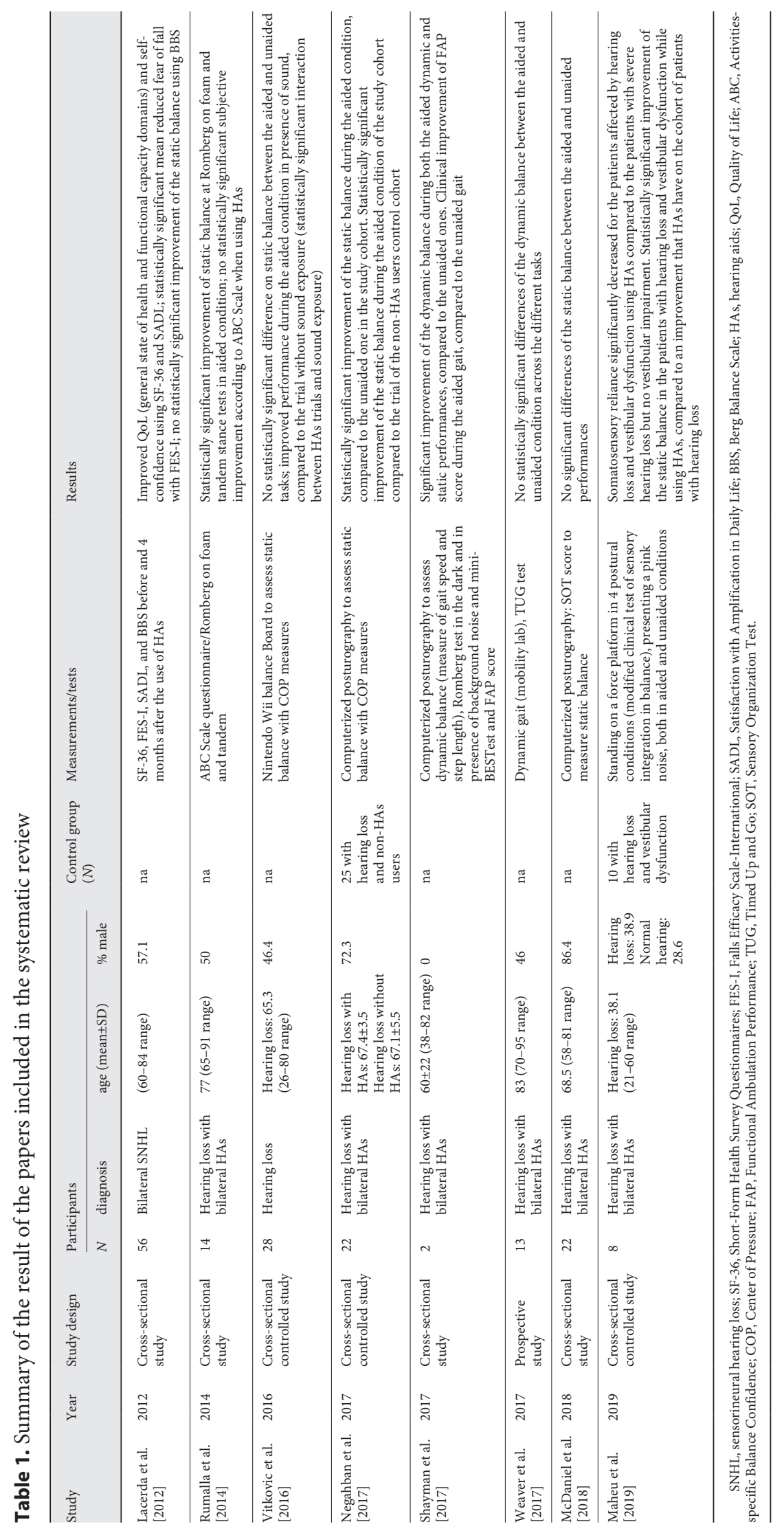




\section{Effect of Hearing Aids on Balance}

\section{Clinical Investigations}

Static Balance

Two studies ( $n=16)$ [Rumalla et al., 2015; Shayman et al., 2017] used the Romberg test (on foam and/or tandem stance) to measure the benefit of HAs on balance. A significant improvement $(p=0.005)$ of the Romberg test performances calculated as the length of time over which subjects could stand (up to a maximum of $30 \mathrm{~s}$ ) during aided conditions compared to the unaided trials was noted [Rumalla et al., 2015]. However, a direct correlation between hearing gain and proportional improvement of the Romberg test performance was not noted. Shayman et al. [2017] observed an improvement from the unaided to aided task in both study participants, though inferential statistical tests were not performed due to the small size of the cohort.

\section{Dynamic Balance}

In one study, FAP was used as a clinical score to evaluate the benefit of HAs on dynamic balance in 2 patients [Shayman et al., 2017]; the authors reported a clinical improvement according to the FAP score during the aided gait (mean FAP score $=78$ ), compared to the unaided gait (mean FAP score $=67.5$ ). However, due to the small size of the cohort, inferential statistics were not performed.

TUG test was used in one study [Weaver et al., 2017], but no significant difference of dynamic balance was found between the aided and the unaided task. Conversely, Mini-BESTest, analogous to the TUG test, was performed in one study in 2 patients [Shayman et al., 2017] and showed an improvement in dynamic balance when patients were wearing HAs, compared to the unaided trial. Statistical tests were not performed, due to the small sample size.

\section{Objective Measurement of Balance}

Static Balance

Computerized posturography was used as objective measurement of static balance in 4 studies [Vitkovic et al., 2016; Negahban et al., 2017; McDaniel et al., 2018; Maheu et al., 2019], but the results are inconsistent. Out of a total of 41 patients with moderate hearing loss, McDaniel et al. [2018] and Vitkovic et al. [2016] did not find any significant difference on static balance between the aided and unaided performances $(p=0.124$ and $p>0.05$, respectively). Nevertheless, Vitkovic et al. [2016] observed an improvement of static balance during the aided condition in presence of sound, compared to the trial without sound

Hearing Aids and Balance Control exposure (significant interaction between HAs trial and sound environment, $p=0.048$ ).

Static balance was noted to be significantly improved by Negahban et al. [2017] on a total of 22 patients with moderate hearing loss, during the aided condition, compared to the unaided $(p<0.0001)$. Furthermore, Negahban et al. [2017] found a statistically significant improvement of the static balance during the aided condition in the group of 22 patients with moderate hearing loss compared to a control cohort of 25 non-HAs users with moderate hearing loss $(p<0.0001)$.

Maheu et al. [2019] enrolled a total of 10 patients affected by severe hearing loss and vestibular dysfunction documented by peripheral vestibular objective measurements, and he found that somatosensory reliance significantly decreased for these 10 participants using HAs compared to 14 participants with normal hearing ( $p=$ 0.009 ) and 8 participants with severe hearing loss but no vestibular impairment $(p=0.008)$. They also documented a statistically significant improvement of the static balance in these 10 patients while using HAs, compared to an improvement that HAs have on the cohort of 8 patients with hearing loss, both in the condition of eyes open and on foam $(p=0.013)$ and of eyes closed and on foam $(p=0.004)$.

\section{Dynamic Balance}

Computerized posturography was used to objectively evaluate ambulation tasks in one study with 2 patients, finding an improvement in balance during the aided dynamic performances, compared to the unaided tests [Shayman et al., 2017]. However, due to the small cohort, no statistical test was performed. Inertial sensor data from 13 patients were analyzed through Mobility Lab to obtain objective measurements of dynamic balance during gait in one study [Weaver et al., 2017], and did not show statistically significant differences of balance between the aided and unaided condition.

\section{Quality of Life}

Only one study [Lacerda et al., 2012] evaluated the QoL $(n=28)$ in patients affected by hearing loss using SF36 and SADL questionnaires. They demonstrated a mean statistically significant improved QoL perception (as general state of health and functional capacity domains) and self-confidence after using HAs for 4 months $(p<0.05)$.

\section{Subjective Measurements of Balance}

Two studies evaluated the effect of HAs on subjective tests [Lacerda et al., 2012; Rumalla et al., 2015]. A signifi- 
cant mean reduced fear of falling perception after using hearing devices for 4 months was reported in 28 patients with hearing loss by using the FES-I questionnaire $(p<$ 0.05 ), while no significant balance improvement was observed by using the BBS $(p>0.05)$ [Lacerda et al., 2012]. By using ABC Scale measurements on a different cohort of 14 patients, the subjective improvement was not statistically significant either [Rumalla et al., 2015].

\section{Adverse Outcomes}

No studies reported any increased rate of adverse events associated with provision of HAs.

\section{Discussion}

\section{Summary of Results}

This systematic review included a total of 200 patients across 8 studies. When using clinical investigations without objective tests, an overall improvement in static balance within aided conditions was noted using the Romberg test [Rumalla et al., 2015; Shayman et al., 2017], while an improvement in dynamic balance using clinical investigations was also noted in patients wearing HAs (FAP and mini-BESTest) but not when measuring using the TUG test [Shayman et al., 2017; Weaver et al., 2017].

Static balance in patients with HAs, measured using computerized posturography, demonstrated an improvement in 1 out of 4 studies compared to unaided conditions [Negahban et al., 2017]. However, no significant difference or worsened static balance were noted in 2 out of 4 studies in aided conditions [Vitkovic et al., 2016; McDaniel et al., 2018], although an improvement of static balance in the aided conditions and in presence of sounds was noted, compared to the trial without sound exposure [Vitkovic et al., 2016]. Only 1 out of 4 studies using computerized posturography while using HAs observed a significant improvement of the static balance in patients affected by hearing loss and vestibular loss, compared to patients affected only by hearing loss [Maheu et al., 2019]. Computerized posturography was also used to analyze dynamic balance in one study, where an improved performance during the aided conditions was documented [Shayman et al., 2017].

Statistically significant differences were noted in dynamic balance between aided and unaided trials through computed posturography [Shayman et al., 2017] but not using inertial sensor measurements with the Mobility Lab device [Weaver et al., 2017], although both studies had small cohorts of patients, 2 and 13, respectively. An im- proved QoL perception and self-confidence was documented in patients with HAs in one study where this outcome was measured [Lacerda et al., 2012]. Subjective measurements of balance were used in 2 studies, with conflicting results, showing a reduced fear of fall perception using HAs only on the FES-I [Lacerda et al., 2012] but no statistically significant improvement in balance with the BBS [Lacerda et al., 2012] or the ABC Scale measurements [Rumalla et al., 2015]. Our analysis found that patients affected by hearing loss can have some benefit in their balance control when using HAs, most of all in the presence of auditory inputs or in the presence of an objective vestibular loss where audiological cues help compensate in maintaining balance.

\section{Hypotheses behind Hearing Loss and Imbalance}

The idea that sound could play a role in balance processes has been promoted since 1985 [Era and Heikkinen 1985; Gandemer et al., 2017], and further other experiences confirmed that hearing loss can be linked to unfavorable postural control [Era and Heikkinen, 1985; Suarez et al., 2007; Mangiore, 2012; Gandemer et al., 2017]. A possible explanation of the influence of auditory inputs on balance involves the concept that sound impulses could represent spatial orienting landmarks. Therefore, the sound localization processing could help the subject to create a 3-dimensional map of the environment, in order to reduce fluctuations in the sway velocity and maintain balance in relation to the sound sources [Rumalla et al., 2015; Vitkovic et al., 2016; Maheu et al., 2019]. In these terms, sound inputs act as audio biofeedback, useful to improve postural corrections in order to maintain balance. This demonstrated proved, for example, in 50 subjects with normal hearing where the effect of sound environment showed a significant improvement on static balance $(p<0.001)$ and a higher sway in normal-hearing subjects was noted in conditions where there were fewer sound cues available [Vitkovic et al., 2016]. This suggests that a spatial hearing map contributes to balance control, although the effect size was considerably smaller than the contribution from the somatosensory and visual systems.

It has also been postulated that in instances of reduced sensory redundancy, for example in patients affected by hearing loss and imbalance, auditory spatial cues could be involved in maintaining balance, and then auditory biofeedback systems could be used as effective adjuvant treatment options in vestibular rehabilitation [Vitkovic et al., 2016; Maheu et al., 2019]. Moreover, Maheu et al. [2019] also proposed another hypothesis to explain the improvement of balance observed in patients affected by 
vestibular impairment wearing HAs, which is the crossmodal stochastic resonance. It was postulated that auditory inputs may enhance the detection of another sensory modality, such as residual somatosensory cues, overall improving balance function. In contrast, the influence of hearing function on dynamic balance has not been clearly assessed [Shayman et al., 2017; Weaver et al., 2017]. It has been theorized that sound inputs may have a major effect on static balance rather than on gait because the localization cues are less consistent during movement due to the constant change of auditory information and therefore it is less integral to maintaining balance.

\section{Comparison with Other Studies}

In patients affected by hearing loss, the role of the auditory amplification in enhancing auditory spatial cues has been investigated primarily in the CI population [Ibrahim et al., 2017], with less prominence and emphasis on patients using HAs. Balance improvement after CI surgery has shown contradictory results [Buchman et al., 2004; Suarez et al., 2007; Kluenter et al., 2009; Huang et al., 2011; Cushing et al., 2012]. Drawing a definitive conclusion about balance gain in patients who underwent a $\mathrm{CI}$ is rather difficult for a number of reasons: (i) adult subjects are largely fitted unilaterally, thereby eliminating any spatial benefits of binaural hearing which could contribute to postural stability; (ii) the underlying etiology of the cochlea loss may also be associated with other vestibular deficits, and there is additional ambiguity regarding inadvertent electrical stimulation of the vestibular nerve via the cochlear implant; (iii) heterogeneity in study design, variability among patient populations, etiology of SNHL (e.g., meningitis often results in disturbed vestibular function due to ossification of the labyrinth [Cushing et al., 2012]), pre-existing conditions, measurement and reporting differences.

Hearing restoration with CI can cause a direct trauma by electrode insertion, acute serous labyrinthitis due to cochleostomy, foreign body reaction with labyrinthitis, endolymphatic hydrops, and electrical stimulation from the implant itself [Katsiari et al., 2013], all of which can cause a variable effect on the vestibular system causing worsening in balance, contrary to rehabilitation using HAs. A deterioration in the non-implanted ear might occur either because the insertion of the electrode in the scala tympani in one ear alters the vestibular input to the brain and hence modifies the contralateral ear response or because the reproducibility of the response in these individuals over time is not perfect [Katsiari et al., 2013; Abramides et al., 2015].

Hearing Aids and Balance Control

\section{Limitations/Bias of Review Process}

Limitations and bias of the review process included (i) data available were mainly provided through descriptive studies with small cohorts of patients; (ii) the lack of blinded, randomized-controlled studies; and (iii) the lack of data analyzing the outcome in balance based on the degree of hearing loss or (iv) based on unilateral VS bilateral hearing loss. When analyzing the data, it is important that these factors are considered.

\section{Applicability of Evidence}

Imbalance and falls are considered by the World Health Organization as an important burden on the global healthcare system [WHO, 2008]. The prevalence of imbalance in individuals aged above 60 years has been documented to be as high as 58\% [Semenov et al., 2016]. Imbalance related to vestibular dysfunction is also associated with a 2.6-fold increase in the odds of falling. Therefore, an increased awareness of the implications of imbalance and understanding of the potential consequences of patients with poorly controlled balance function is vital. Even if the overall contribution of HAs to the maintenance of balance has not been well defined in the literature, according to this systematic review, it is possible to assess that improved auditory inputs could contribute to balance control due to different factors: (i) auditory cues are normally used by subjects with normal hearing to improve balance; (ii) an objective improvement in maintaining balance is obtained in patients affected by SNHL wearing HAs [Vitkovic et al., 2016; Negahban et al., 2017; Shayman et al., 2017], suggesting that HAs may provide renewed access to spatial cues, thus improving balance control.

HAs are generally very well tolerated with a low rate of adverse events [Ferguson et al., 2017] and therefore it is worth considering auditory rehabilitation in such patient groups to not only improve their auditory function but also potentially improve patients' static balance and selfconfidence. It is worth noting that the varied balance outcomes could potentially be explained through the different sound processing algorithms used by HAs, which may affect and/or distort the potential amplification of spatial cues in various ways [Vitkovic et al., 2016]. Additionally, the degree of hearing loss and the frequency range involved have also been noted as possible additional factors that may affect balance outcomes.

\section{Future Research}

In this review, we highlighted the lack of high-quality evidence on this topic. Further trials should focus on areas with lack of evidence of effect and prospective, ran- 
domized studies should be undertaken to explore the benefit of HAs, in people affected from hearing loss, both in static and dynamic balance. Future research should take into account variables such as the age, the degree of hearing loss, the wide array of hearing loss phenotypes, the differing hearing-aid devices available for auditory rehabilitation and if differences exist between unilateral and bilateral hearing loss, when standardizing results.

\section{Conclusion}

Our study suggests an improvement in balance in patients with HAs under certain conditions. However, due to the unclear correlation between subjective and objective measures of balance in patients wearing HAs, it is only possible to speculate that HAs may also improve static, dynamic, or subjective perception of balance function in adults affected by hearing loss.

\section{Acknowledgement}

The Authors thank Jacqueline Smith, clinical librarian at UCL Library for her professional support during the systematic review search.

\section{Statement of Ethics}

No ethical approval will be needed because data from previous published studies in which informed consent was obtained by primary investigators will be retrieved and analyzed.

\section{Conflict of Interest Statement}

The authors have no conflicts of interest to declare.

\section{Funding Sources}

No funding was received to conduct the research shown in the manuscript.

\section{Author Contributions}

Daniele Borsetto and Virginia Corazzi: contributed substantially to the conception and the interpretation of the data for the work, drafted the work, final approved the version to be published, and are accountable for all aspects of the work in ensuring that questions related to the accuracy or integrity of any part of the work are appropriately investigated and resolved.

Sebastiano Franchella, Chiara Bianchini, Stefano Pelucchi, and Andrea Ciorba: contributed substantially to design the work, to the acquisition, analysis of data for the work, revised it critically for important intellectual content, final approved of the version to be published, and are accountable for all aspects of the work in ensuring that questions related to the accuracy or integrity of any part of the work are appropriately investigated and resolved.

Rupert Obholzera, Andrew J. Soulbya, and Nikul Amina: contributed substantially to the design of the work, acquisition and interpretation of data for the work, revised it critically for important intellectual content, final approved the version to be published, and are accountable for all aspects of the work in ensuring that questions related to the accuracy or integrity of any part of the work are appropriately investigated and resolved.

\section{References}

Abramides PA, Bittar RS, Tsuji RK, Bento RF. Caloric test as a predictor tool of postural control in CI users. Acta Otolaryngol. 2015;135(7): 685-91.

Access Economics. Listen, Hear! The Economic Impact and Cost of Hearing Loss in Australia. Boca Raton,FL: CRC; 2006.

Buchman CA, Joy J, Hodges A, Telischi FF, Balkany TJ. Vestibular effects of cochlear implantation. Laryngoscope. 2004;114(10 Pt 2 Suppl 103):1-22.

Callisaya ML, Blizzard L, McGinley JL, Srikanth VK. Risk of falls in older people during fastwalking: the TASCOG study. Gait Posture. 2012;36(3):510-5.

Cushing SL, Pothier D, Hughes C, Hubbard BJ, Gordon KA, Papsin BC. Providing auditory cues to improve stability in children who are deaf. Laryngoscope. 2012;122(Suppl 4):S101-2.
Deal JA, Sharrett AR, Albert MS, Coresh J, Mosley TH, Knopman D, et al. Hearing impairment and cognitive decline: a pilot study conducted within the atherosclerosis risk in communities neurocognitive study. Am J Epidemiol. 2015;181(9):680-90.

Era P, Heikkinen E. Postural sway during standing and unexpected disturbance of balance in random samples of men of different ages. J Gerontol. 1985;40(3):287-95.

EuroStat [Internet]. 2015 [cited 2020 May 29]. Available from: http://ec.europa.eu/eurostat. Ferguson MA, Kitterick PT, Chong LY, Edmondson-Jones M, Barker F, Hoare DJ. Hearing aids for mild to moderate hearing loss in adults. Cochrane Database Syst Rev. 2017; 9(9):CD012023.
Gandemer L, Parseihian G, Kronland-Martinet R, Bourdin C. Spatial cues provided by sound improve postural stabilization: evidence of a spatial auditory map? Front Neurosci. 2017; 11:357.

Gates GA, Gibbons LE, McCurry SM, McCusrry SM, Crane PK, Feeney MP, et al. Executive dysfunction and presbycusis in older persons with and without memory loss and dementia. Cogn Behav Neurol. 2010;23(4):218-23.

Hogan A, O'Loughlin K, Miller P, Kendig H. The health impact of a hearing disability on older people in Australia. J Aging Health. 2009; 21(8):1098-111.

Huang CQ, Dong BR, Lu ZC, Yue JR, Liu QX. Chronic diseases and risk for depression in old age: a meta-analysis of published literature. Ageing Res Rev. 2010;9(2):131-41. 
Huang MW, Hsu CJ, Kuan CC, Chang WH. Static balance function in children with cochlear implants. Int J Pediatr Otorhinolaryngol. 2011;75(5):700-3.

Ibrahim I, da Silva SD, Segal B, Zeitouni A. Effect of cochlear implant surgery on vestibular function: meta-analysis study. J Otolaryngol Head Neck Surg. 2017;46(1):44.

Katsiari E, Balatsouras DG, Sengas J, Riga M, Korres GS, Xenelis J. Influence of cochlear implantation on the vestibular function. Eur Arch Otorhinolaryngol. 2013;270(2):489-95.

Kluenter HD, Lang-Roth R, Guntinas-Lichius O. Static and dynamic postural control before and after cochlear implantation in adult patients. Eur Arch Otorhinolaryngol. 2009; 266(10):1521-5.

Lacerda CF, Silva LO, de Tavares Canto RS, Cheik NC. Effects of hearing aids in the balance, quality of life and fear to fall in elderly people with sensorineural hearing loss. Int Arch Otorhinolaryngol. 2012;16(2):156-62.

Loughrey DG, Kelly ME, Kelley GA, Brennan S, Lawlor BA. Association of age-related hearing loss with cognitive function, cognitive impairment, and dementia: a systematic review and meta-analysis. JAMA Otolaryngol Head Neck Surg. 2018;144(2):115-26.

Lord SR, Menz HB, Tiedemann A. A physiological profile approach to falls risk assessment and prevention. Phys Ther. 2003;83(3):23752.

Maheu M, Behtani L, Nooristani M, Houde MS, Delcenserie A, Leroux T, et al. Vestibular function modulates the benefit of hearing aids in people with hearing loss during static postural control. Ear Hear. 2019;40(6):1418-24.

Mangiore RJ. The effect of an external auditory stimulus on postural stability of participants with cochlear implants.
McDaniel DM, Motts SD, Neeley RA. Effects of bilateral hearing aid use on balance in experienced adult hearing aid users. Am J Audiol. 2018;27(1):121.

Mick P, Parfyonov M, Wittich W, Phillips N, Guthrie D, Kathleen Pichora-Fuller M. Associations between sensory loss and social networks, participation, support, and loneliness: analysis of the Canadian Longitudinal Study on Aging. Can Fam Physician. 2018;64(1): e33-41.

Moher D, Shamseer L, Clarke M, Ghersi D, Liberati A, Petticrew M, et al. Preferred reporting items for systematic review and meta-analysis protocols (PRISMA-P) 2015 statement. Syst Rev. 2015;4(1):1.

Negahban H, Bavarsad Cheshmeh Ali M, Nassadj G. Effect of hearing aids on static balance function in elderly with hearing loss. Gait Posture. 2017;58:126-9.

Piirtola M, Era P. Force platform measurements as predictors of falls among older people: a review. Gerontology. 2006;52(1):1-16.

Rumalla K, Karim AM, Hullar TE. The effect of hearing aids on postural stability. Laryngoscope. 2015;125(3):720-3.

Semenov YR, Bigelow RT, Xue QL, du Lac S, Agrawal Y. Association between vestibular and cognitive function in U.S. adults: data from the National Health and Nutrition Examination Survey. J Gerontol A Biol Sci Med Sci. 2016;71(2):243-50.

Shayman CS, Earhart GM, Hullar TE. Improvements in gait with hearing aids and cochlear implants. Otol Neurotol. 2017;38(4):484-6.

Suarez H, Angeli S, Suarez A, Rosales B, Carrera $\mathrm{X}$, Alonso R. Balance sensory organization in children with profound hearing loss and cochlear implants. Int J Pediatr Otorhinolaryngol. 2007;71(4):629-37.
Swanenburg J, de Bruin ED, Uebelhart D, Mulder T. Falls prediction in elderly people: a 1-year prospective study. Gait Posture. 2010;31(3): $317-21$

Taljaard DS, Olaithe M, Brennan-Jones CG, Eikelboom RH, Bucks RS. The relationship between hearing impairment and cognitive function: a meta-analysis in adults. Clin Otolaryngol. 2016;41(6):718-29.

Thaler-Kall K, Peters A, Thorand B, Grill E, Autenrieth CS, Horsch A, et al. Description of spatio-temporal gait parameters in elderly people and their association with history of falls: results of the population-based crosssectional KORA-Age study. BMC Geriatr. 2015;15:32.

Verghese J, Holtzer R, Lipton RB, Wang C. Quantitative gait markers and incident fall risk in older adults. J Gerontol A Biol Sci Med Sci. 2009;64(8):896-901.

Viljanen A, Kaprio J, Pyykkö I, Sorri M, Pajala S, Kauppinen M, et al. Hearing as a predictor of falls and postural balance in older female twins. J Gerontol A Biol Sci Med Sci. 2009; 64(2):312-7

Vitkovic J, Le C, Lee SL, Clark RA. The contribution of hearing and hearing loss to balance control. Audiol Neurootol. 2016;21(4):195202.

Weaver TS, Shayman CS, Hullar TE. The effect of hearing aids and cochlear implants on balance during gait. Otol Neurotol. 2017;38(9): 1327-32.

WHO Global Report on Falls Prevention in Older Age. Geneva, Switzerland: World Health Organization Press [Internet] 2008 [cited 2020 Jun 24]. Available from: http://www.who.int/ ageing/publications/Falls_prevention7March.pdf. 